\title{
Long Time Pulse Compression Method Based on Coherent Integration
}

\author{
Baisen Liü, 2, a *, Ye Zhang, b and Wulin Zhang ${ }^{3, c}$ \\ ${ }^{1}$ School of Electronics and Information Engineering, Harbin Institute of Technology, Harbin, China \\ ${ }^{2}$ School of Electrical and Information Engineering, Heilongjiang Institute of Technology, Harbin, China \\ ${ }^{3}$ School of Information and Communication Engineering, Harbin Engineering University, Harbin, China \\ asped_liu@126.com, b'zhangye@hit.edu.cn, '240577393@qq.com
}

Keywords: MIMO radar; Coherent integration; Pulse compression; Signal separation

\begin{abstract}
MIMO radar signals emitted from the transmitting side are not the same, the signal received by the receiver is the sum of each emission signal, Therefore, and we need to separate the signal to waveform transmitted by MIMO radar at the receiving end. Adaptive pulse compression method based on iteration can effectively separate MIMO radar transmitting signal, however, this method is adaptive weighting the received signal, through several times of iteration computation, to obtain the best value, so that we can carry out pulse compression. Since the noise is random, each weighted value of the received signal is different. Therefore, we cannot carry out coherently accumulated pulse. To solve this problem, in this paper, we have established a model which MIMO radar receives signal through pulse coherent accumulation mode, and put forward a kind of pulse compression method based on linear constrained minimum variance. This method does not require multi-iterations and easy to calculate. In this way, we can carry out coherently accumulated pulse. The simulation results show that this method can effectively separate MIMO radar signal.
\end{abstract}

\section{Introduction}

MIMO radar can form a low gain wide beam by emitting a set of orthogonal waveforms [1]. The receiver separates signal through a set of matched filter bank which is suitable for different emission signals. Then digital signal processes whose target detection performance and low probability of intercept capability superior to conventional radar [2,3]. MIMO radar requires transmitting waveforms are orthogonal waveforms. However, there is no completely orthogonal waveform. Thus, the cross-correlation between the transmitted signal, the radar echo signal not only inevitably lead to the presence of noise superposition,but also there is mutual interference between the echo signal, thereby side lobes are improved and this affects the detection performance of targets. Therefore, in order to improve the signal separation, design a set of compression filter which can effectively separate pulse is necessary.

If the MIMO radar irradiation the target for a long time, what's more it is coherent, it can improve the accumulated output SNR by coherent integration and can obtain better Doppler resolution [4]. In order to effectively separate the signal at the receiving end, adaptive Pulse compression methods based on the minimum mean square error criteria and guidelines to maximize the signal to noise ratio are capable of suppressing the signal from the side lobe itself, reduce the level of cross-correlation between the signals, better solve the problem of mutual interference between the various transmit waveforms. However, these two methods is adaptively weighted reception signals. Through several times of iteration, we can get the optimal weights for pulse compression. Since the noise is random, the weighted value of each received signal is completely different. Therefore, even if the coherent burst is emitted, different weights will destroy the coherent nature this is not conducive to improving the signal to noise ratio which has been accumulated for a long time. To solve this problem, according to linearly constrained minimum variance criterion, based on long coherent integration, a kind of pulse 
compression method has been proposed.

\section{MIMO Radar Receiving Signal Model}

Let us suppose that MIMO radar work under the condition of narrowband far field, receiving array and transmitting array are in the same phase center [5]. It is composed of $M$ transmit antennas and $N$ reception antennas. For far target sites, when the Doa Angle is $\theta$, the transmit signal steering vector is $a(\theta)=\left[a_{1}(\theta), a_{2}(\theta), \cdots, a_{M}(\theta)\right]^{T} \quad$ Target steering vector of the received signal is $b(\theta)=\left[b_{1}(\theta), b_{2}(\theta), \cdots, b_{N}(\theta)\right]^{T}, \mathrm{M}$ emitting array element transmit signal is $S=\left[s_{1}, s_{2}, \cdots, s_{M}\right]$, among them, $s_{m}=\left[s_{m}(0), s_{m}(1), \cdots, s_{m}(P)\right]^{T}, m=1,2, \cdots M, P$ is the transmitted signal sampling points, the received signal is $r(l)=\left[r_{1}(l), r_{2}(l), \cdots, r_{N}(l)\right], l=1,2, \cdots, L$.Scattering intensity of single target in space is $\alpha, e_{n}$ is the noise, Thus, we can get the signal received by the $n$-th array element antenna at the time of $l$ :

$$
r_{n}(l)=\alpha b_{n}(\theta) S(l) a(\theta)+e_{n}(l)
$$

The NO $L$ continuous time samples at the $l$-th moment is:

$$
r_{n}(l)=\left[r_{n}(l), r_{n}(l+1), \cdots, r_{n}(l+P-1)\right]^{T}
$$

According to the working principle of MIMO radar and signal processing flow, each signal received by each array element matched filtering with $M$ transmit signal. The expressions after signal received by $n$-th array element matched with $m$-th emission signal are as follows:

$$
\begin{aligned}
y_{n m}(l) & =s_{m}^{H}(l) r_{n}(l)=\alpha b_{n}(\theta) s_{m}{ }^{H}(l) S[l] a(\theta)+s_{m}{ }^{H}(l) e_{n}(l) \\
& =\alpha b_{n}(\theta) a_{m}(\theta) s_{m}{ }^{H}(l) s_{m}(l)+\alpha b_{n}(\theta) * \sum_{q \neq m}{ }^{M} a_{q}(\theta) s_{q}{ }^{H}(l) s_{q}(l)+s_{m}{ }^{H}(l) e_{n}(l)
\end{aligned}
$$

In the formula (3), The first item is the $m$-th autocorrelation component of the transmitted signal. The second item is the sum of the $m$-th transmitted signal and other components of the cross-correlation of the transmitted signal. The third item is the noise component. Thus, the output of filter contains not only the autocorrelation of the signal itself, but cross-correlation component between signals and related components of transmit signal and noise as well.

\section{Pulse Compression Method Based on Long Time Coherent Integration}

MIMO Radar Receiving Signal Model. Let us suppose that MIMO radar work under the condition of narrowband far field, receiving array and transmitting array are in the same phase center, It is composed of $M$ transmit antennas and $N$ reception antennas, coherent pulse series contains $K$ coherent pulse. For far target site, when the Doa Angle is $\theta$, the transmit signal steering vector is $a(\theta)=\left[a_{1}(\theta), a_{2}(\theta), \cdots, a_{M}(\theta)\right]^{T}$, Target steering vector of the received signal is $b(\theta)=\left[b_{1}(\theta), b_{2}(\theta), \cdots, b_{N}(\theta)\right]^{T}, \mathrm{M}$ emitting array element transmit signal is $S=\left[s_{1}, s_{2}, \cdots, s_{M}\right]$, among them, $s_{m}=\left[s_{m}(0), s_{m}(1), \cdots, s_{m}(P)\right]^{T}, \quad m=1,2, \cdots M, P$ is the transmitted signal sampling points, each sampling point corresponds to a distance unit, the received signal is $r(l)=\left[r_{1}(l), r_{2}(l), \cdots, r_{N}(l)\right]$, $l=1,2, \cdots, L$.Scattering intensity of single target in space is $\alpha, e_{n}$ is the noise. Thus, we can get the $k_{-t h}$ 


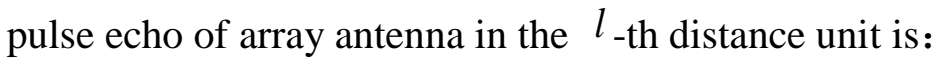

$$
r_{n}^{k}(l)=\alpha b_{n}(\theta) S(l) a(\theta)+e_{n}^{k}(l)
$$

Then $P \times K$ observation echo matrix which is made up of $K$ pulses and $P$ distance units is:

$$
Y(l)=\left[\begin{array}{ccc}
r_{n}^{1}(l) & \cdots & r_{n}^{K}(l) \\
\vdots & & \vdots \\
r_{n}^{1}(l+(P-1)) & \cdots & r_{n}^{K}(l+(P-1))
\end{array}\right]
$$

Pulse Compression Method. Considering pulse compression of coherent integration, in order to achieve effective suppression to the interference between the signals brought by transmitted waveform cross-correlation and separate the $m$-th transmission signal received by the $n$-th receiving antenna of $K$ pulses, to maintain energy of a scattering point of a certain distance unit- $l$-constant and minimize the energy from other distance unit, mathematically expressed as follows:

Among them, $h_{n m}(l)$ is the weight coefficient of the $m$-th filter on the $n$-th receiving array element, $g=[1,0, \cdots, 0]^{T} S=\left[s_{m}, s_{1}, \cdots, s_{M}\right], R_{n}(l)$ is the covariance matrix of observations echo Matrix [6,7]. The mathematical expression of $R_{n}(l)$ is:

$$
R_{n}(l)=\frac{Y(l) Y^{H}(l)}{P K}
$$

Solving method of the optimal weight vector in formula (6) is the same as that in Capon Beam-forming Algorithm. We can solve this problem by Lagrange multipliers. Optimal weight vector $h_{n m}(l)$-is:

$$
h_{n m}(l)=\left[R_{n}(l)\right]^{-1} S\left[S^{H} R_{n}(l) S\right]^{-1} g
$$

The output of pulse compression filter based on long time coherent integration is:

$$
y_{n m}(l)=\left[\left[R_{n}(l)\right]^{-1} S\left[S^{H} R_{n}(l) S\right]^{-1} g\right]^{H} Y(l)
$$

The whole algorithm consists of the following steps:

According to the data structure of the $K$-th pulse-echo observe echo matrix $Y(l)$

Calculate the covariance matrix, $R_{n}(l)$, of $Y(l)$

Put $R_{n}(l)$ into the formula (7), get the optimal weight vector $h_{n m}(l)$.Put $h_{m}(l)$ into the formula (8). The output of filter is $y_{m}(l)$.

This method has the following features:

This method is based on linearly constrained minimum variance criterion. Use L range cells sampling point to realize adaptive pulse compression processing. Compared with the methods in the literature [8] and the literature [9], the methods in this article are non-iterative. We need to get P-dimensional matrix inversion. Iterative adaptive pulse compression method requires $(2 K-1)(P-1)+1$ distance unit. Each iteration inverses the $P$-dimensional matrix. With the increase of the number $K$ of iterations, the number of distance units to be calculated and the amount of computation greatly 
increased. However, the proposed method of $\mathrm{N}$ pulses requires only $P \times K$ distance unit and only once inverse operation. In this way, we can complete adaptive pulse compression.

This method seeking weight vector by constructing echo the observation matrix to a receiving antenna $K$ pulses, make the weight vector acting on the observation matrix $Y(l)$. We'll get $\mathrm{N}$-dimensional vector which is still coherent between elements. Coherent relationship is decided by the antenna direction vector. So we can use coherent integration to accumulate the energy of the target. This effectively avoids the loss of energy targets, but for the method of adaptive pulse compression which uses a single pulse to observe data and then form a right vector, even if what was emitted is $K$ coherent pulse train, however, due to its weight vector of different pulses will not be identical, it will no longer be coherent between $K$ values obtained by filtering through pulse compression method based on minimum mean square error and the maximum signal to noise ratio. Thus in the subsequent coherent treatment, it will inevitably lead to the loss of energy of the target.

\section{Simulation and Analysis}

Let us assume that the number of received array elements is 4 . The element distance between emitting array element and the array element is half wavelength. We chose four-phase codes signals whose code length is 40 and pulse train contains 50 coherent pulses as transmitted waveform which was designed in the document [10].

In Fig. 1 - Fig. 2, when the SNR is $0 \mathrm{~dB}$ and $15 \mathrm{~dB}$, we can see the four signals which the first receiving antenna corresponding to the first pulse in the pulse train. It uses the proposed method and the conventional matched filter to compress separated pulse and then output. Fig. 1. and Fig. 2 shows that the proposed method has a good result of signal separation when the echo is single. When the SNR is $0 \mathrm{~dB}$, after $s_{1}$ was separated by the two methods, the maximum distance side lobe were $-4.6 \mathrm{~dB}$ and $-17.3 \mathrm{~dB}$. After $s_{2}$ was separated, the maximum distance side lobe were $-7.4 \mathrm{~dB}$ and $-16.8 \mathrm{~dB}$. After $s_{3}$ was separated, the maximum distance side lobe were $-3.2 \mathrm{~dB}$ and $-19.4 \mathrm{~dB}$. After $s_{4}$ was separated by the two methods, the maximum distance side lobe were $-7.1 \mathrm{~dB}$ and $-13.5 \mathrm{~dB}$. When the SNR is $15 \mathrm{~dB}$, after $s_{1}$ was separated by the two methods, the maximum distance side lobe were $-7.3 \mathrm{~dB}$ and $-25.4 \mathrm{~dB}$. After $s_{2}$ was separated, the maximum distance side lobe were $-7.6 \mathrm{~dB}$ and $-25.4 \mathrm{~dB}$. After $s_{3}$ was separated, the maximum distance side lobe were $-4.9 \mathrm{~dB}$ and $-27.3 \mathrm{~dB}$. After $s_{4}$ was separated by the two methods, the maximum distance side lobe were $-7.3 \mathrm{~dB}$ and $-26.7 \mathrm{~dB}$. Compared with the traditional matched filter, the proposed method can largely suppress the range side lobe and separate signals effectively.

Fig. 3 is the output of a long time coherent integration pulse compression, when the signal to noise ratio is $0 \mathrm{~dB}$. We can see the four signals which the first receiving antenna corresponding to the pulse train, it uses the proposed method and the conventional matched filter to compress separated pulse and then output. It is revealed by the comparison that after long time coherent integration, sidelobes outputted by filter is greatly reduced. The maximum sidelobes outputted are below - $30 \mathrm{~dB}$. This is far superior to the output of traditional matched filter. This is because after the coherent integration, the output signal to noise ratio was increased, the performance of signal separation is improved. 


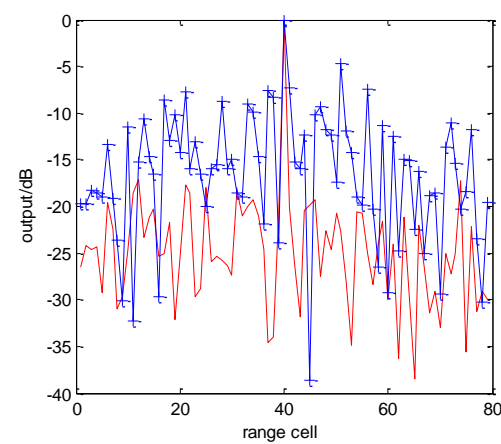

(a) $s_{1}$

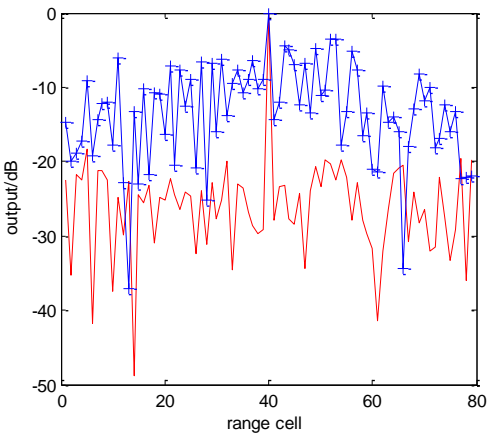

(c) $s_{3}$

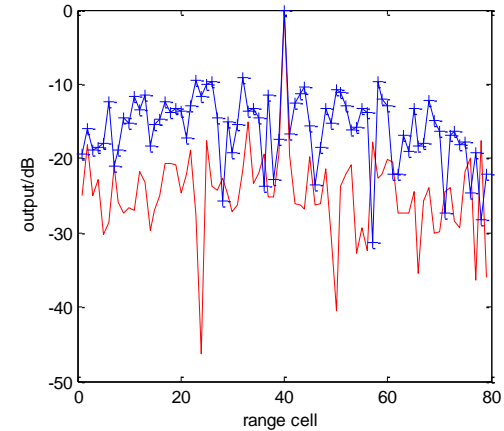

(b) $s_{2}$

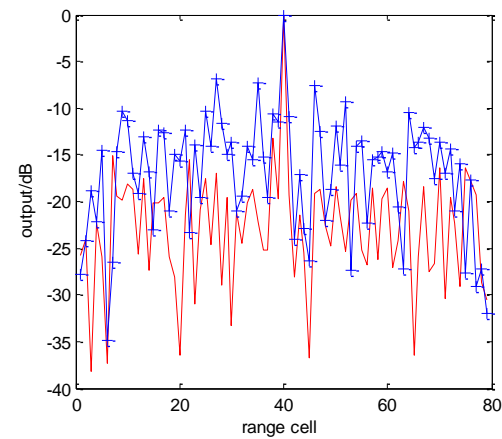

(d) $s_{4}$

-+-+-MF,- - the proposed method

Figure 1. Pulse compression output with $0 \mathrm{~dB}$

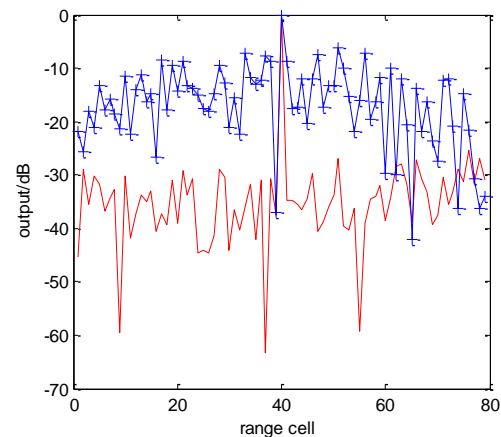

(a) $s_{1}$

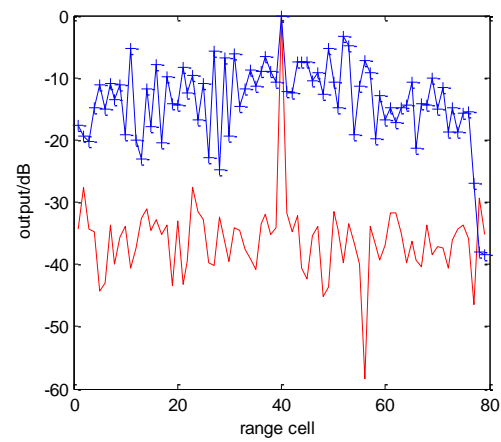

(c) $s_{3}$

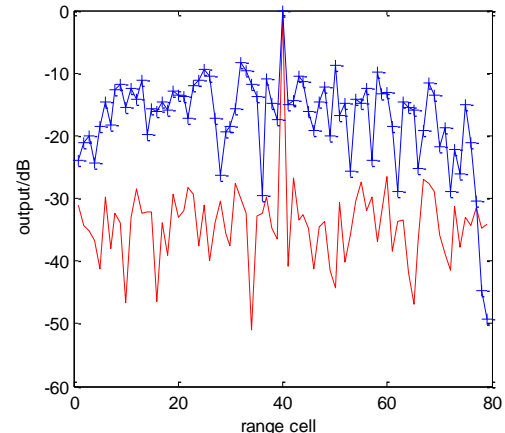

(b) $s_{2}$

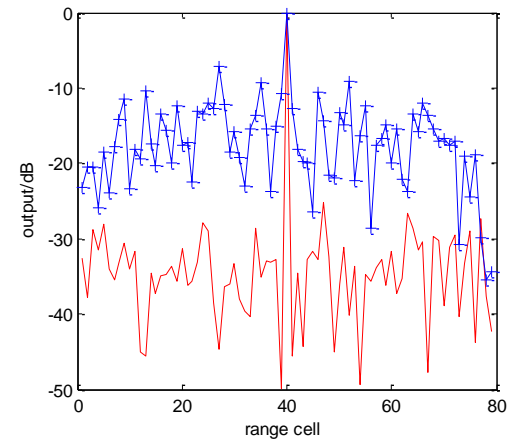

(d) $s_{4}$

-+-+-MF,- - the proposed method

Figure 2. Pulse compression output with $15 \mathrm{~dB}$ 


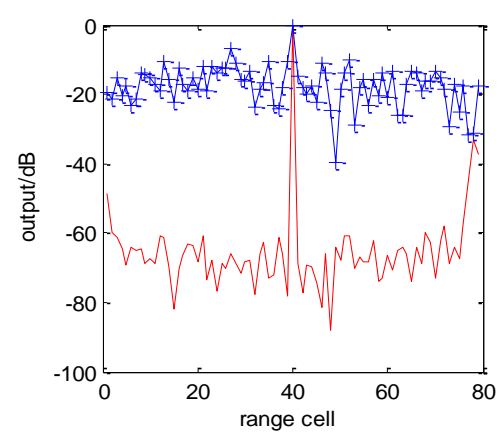

(a) $s_{1}$

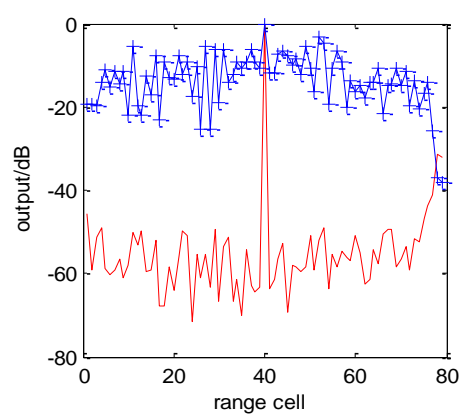

(c) $s_{3}$

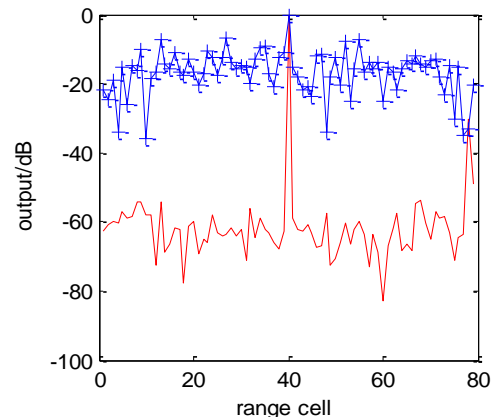

(b) $s_{2}$

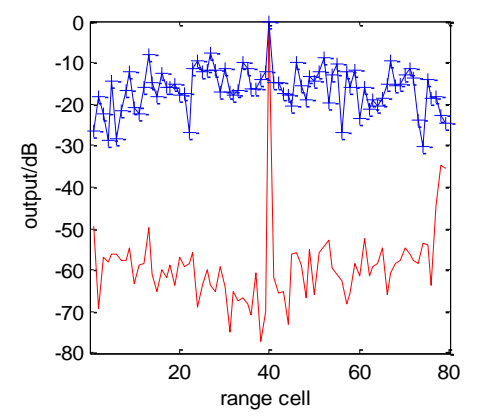

(d) $s_{4}$

-+-+-MF,- - the proposed method

Figure 3. Pulse compression output with $0 \mathrm{~dB}$

\section{Summary}

Effective separation of signals which is transmitted by MIMO radar is one of the keys to realize the target detection of MIMO radar. It is also the basic of detection performance at which MIMO radar is better than conventional radar and radar arrays. In order to solve the problem of by using iterative adaptive pulse compression signal separation method, we cannot realize pulse coherent integration, we propose a method of pulse compression based on linearly constrained minimum variance long time coherent integration. This method has many advantages, for instance, it does not require multi-iterations, it is easy to calculate and it does not destroy the phase coherence between pulses. The simulation results show that this method can effectively separate MIMO radar signal.

\section{Acknowledgements}

This work is supported by Natural Science Foundation of Heilongjiang Province (No. F201322).

\section{References}

[1] H A Khan. Ultra wideband Multiple-Input Multiple-Output radar [C] 2005 IEEE International Radar Conference Arlington, Virginia, 2005, 900- 904.

[2] Jian Li, Petre Stoica, Luzhou Xu, William Roberts. On Parameters Identifiability of MIMO radar [J] IEEE Signal Processing Letters. December 2007, 14(12):968-971. 
[3] Bliss D W, Forsythe K W. Multiple- Input Multiple-Output (MIMO) Radar and Imaging: Degrees of Freedom and Resolution[C], Conference Record of the 37th Asilomar Conference on Signals, Systems and Computers. 2003 Vo1.1:54- 59.

[4] Nikolaus Lehmann, Eran Fishier, Alex Haimovich, eta1 Evaluation of transmit diversity in MIMO radar direction finding[C], IEEE Transaction on Signal Processing, 2007, V1.55 (5):2215-2225.

[5] E Fishier, et a1. MIMO radar: An idea whose time has come[C]. Proceeding of the IEEE Radar Conference. Philadelphia, PA, 2004.71-78.

[6] Huang F, Sheng W, Ma x F. Modified projection approach for robust adaptive array beamforming[J]. Signal Processing, 2012(92): 1758-1763.

[7] Beck A, Eldar Y c. Doubly constrained robust capon beamformer with ellipsoidal uncertainty sets[J]. IEEE Transactions on Signal Processing, 2007, 55(2): 753-758.

[8] Deng H. Polyphase code design for orthogonal netted radar systems [J]. IEEE Trans. on Signal Processing, 2004, 52(11): 3125-3135.

[9] Zhang Juan, Zhang Lin-rang.A Efficient Approach for Adaptive Pulse Compression of MIMO Radar[J]. Journal of Electronics \& Information Technology,2010(1): 17- 21.

[10]LIU Yunfo, LIU Zheng.Adaptive pulse compression for MIMO radar in cross correlation interference [J]. Journal of Xidian University, 2011 (1):89-94. 